\title{
THE
}

6-15-1995

\section{Transport in Channels and Films with Rough Surfaces}

\author{
A. E. Meyerovich \\ University of Rhode Island, sfo101@uri.edu \\ S. Stepaniants \\ University of Rhode Island
}

Follow this and additional works at: https://digitalcommons.uri.edu/phys_facpubs

Terms of Use

All rights reserved under copyright.

\section{Citation/Publisher Attribution}

Meyerovich, A. E., \& Stepaniants, S. (1995). Transport in Channels and Films with Rough Surfaces.

Phys.Rev. B., 51(23), 17116-17130. doi: 10.1103/PhysRevB.51.17116

Available at: http://dx.doi.org/10.1103/PhysRevB.51.17116

This Article is brought to you for free and open access by the Physics at DigitalCommons@URI. It has been accepted for inclusion in Physics Faculty Publications by an authorized administrator of DigitalCommons@URI. For more information, please contact digitalcommons-group@uri.edu. 


\title{
Transport in channels and films with rough surfaces
}

\author{
A.E. Meyerovich and S. Stepaniants \\ Department of Physics, University of Rhode Island, Kingston, Rhode Island 02881
}

(Received 29 December 1994)

\begin{abstract}
We present a simple and versatile description of transport of almost ballistic particles near rough boundaries with an emphasis on thin films and narrow channels. The main effects are associated with chaotization of motion as a result of repeated scattering from random walls. We show that the problem contains an additional mesoscopic length scale which is expressed explicitly via the amplitude and correlation radius (or the correlation function) of surface inhomogeneities, and the ratio of the particle wavelength to the correlation radius. The calculations are performed with the help of a canonical coordinate transformation which reduces a transport problem with rough random walls to a completely equivalent problem with ideal flat walls, but with some random bulk distortions. This problem is treated on the basis of a kinetic equation with a perturbative collision integral. In addition to the application of the Boltzmann transport equation for (quasi) particles with an arbitrary degree of degeneracy of the distribution function, we also include the results for a single-particle diffusion on the basis of the Focker-Plank equation. We calculate different transport coefficients for (quasi) particles with an arbitrary spectrum $\epsilon$ (p) with a bulk of calculations for particles with quadratic, $p^{2} / 2 m$, and linear, $c p$, spectra. The calculations are made in classic and $\mathrm{WKB}$ regimes as well as in the case of quantized motion across the film. All the transport coefficients are expressed via the first two angular harmonics of the correlation function of surface inhomogeneities which play the role of an effective transport cross section. The results include the effects of bulk impurities and changes in potential relief near the walls. We also calculate the quantum interference corrections to conductivity and localization and mesoscopic effects associated with refelections from random surface inhomogeneities, and the density of states in low-dimensional films. The mesoscopic properties are especially simple in the case of strong quantization of motion across the $d$-dimensional films when the problem becomes effectively equivalent to localization of $d$-1-dimensional motion in weak random potential. We discuss possible future applications of our method such as for porous media, boundary slip, etc.
\end{abstract}

\section{INTRODUCTION}

The effects of boundary scattering on transport processes is important for physics of films, waves, and particles propagation in restricted geometry, including the porous media, properties of multilayer systems, quantum wires, etc. These effects become very convoluted if the boundaries are rough with complicated or random inhomogeneities of different scales. In general, the effect of boundary roughness on boundary scattering should lead to chaotization of motion near the boundaries and to some additional diffusion along the walls. It is quite obvious that this transport effect should be described by the correlation function of surface inhomogeneities, but it is not clear how to get such a description in a consistent though simple way. This is the main goal of this paper. Below, we will develop a simple and uniform formalism which can be easily applied to a broad variety of transport problems in very different systems.

The description of transport near rough walls is hindered by two problems. The first one is very important for solid or liquid films, and is less important for gaseous or vacuum systems. In solid or liquid systems, the energy spectrum, including the potential relief and solid-state energy bands, change significantly near the walls. This effect exists even in the case of perfect flat walls. The effects of this type dominate the boundary scattering in many metals or semiconductors, especially in the presence of surface energy levels and/or strong boundary adsorption, when the particle energy experiences dramatic changes near the surface.

The second group of effects is associated directly with the surface roughness, i.e., with the randomness in the exact position and the direction of the boundary which leads to the randomness in phases and directions of reflected particles. In many problems of wave propagation, gas dynamics, electrons in simple metals, etc., the energy changes near the surface play the secondary role with respect to the effects of surface roughness.

These two parts of the scattering problem can often be studied separately, one after another. We will start from the effects of surface roughness, and will address some aspects of energy distortion at the surface closer to the end of this paper.

The scattering from rough walls contributes to the chaotization of motion only as a result of repeated collisions with the walls. The particles can return to the wall either as a result of bulk scattering, or after collisions with another wall. As we will see below, the results for these too cases are different from each other. 
In this paper, we will consider mostly the case of restricted geometry, i.e., narrow channels and thin films, or almost ballistic particles in more bulk systems. We will also point out the necessary changes associated with bulk scattering.

The usual approach to boundary problems is to apply some "exact" boundary condition to an already known bulk problem. Then the scattering by rough walls is described either with the help of an over-complicated boundary condition (see, e.g., Refs. 1-7), which leads to a practically unsolvable integrodifferential transport problem, or by an over-simplified phenomenological boundary condition, which balances partially specular and partially diffuse reflection. The standard perturbative methods ${ }^{3,6,8}$ are also not very useful, especially for electron problems.

A different approach ${ }^{9}$ is to substitute the surface roughness by some phenomenological bulk potential (or, what is very similar, by a set of scattering centers ${ }^{10}$ ) near the surface, and to express the transport characteristics near the surface via the parameters of this potential. This approach can reproduce certain important features of transport processes, including quadratic dependence of conductivity on film thickness, longrange correlations, ${ }^{11}$ and spin exchange processes in multilayers ${ }^{12,13}$ by expressing transport parameters via the correlation function of this phenomenological potential. However, though it was clear ${ }^{9,11}$ that the effective potential is related to the shape of the surface, the explicit form of this relation and, therefore, the form of the effective potential remained unknown and could not be easily reconstructed either theoretically or from experiment. Therefore, following Ref. 9, the calculations were restricted to the simplest model forms of such a potential or its correlation function. Meanwhile, the consistent comparison with experimental data requires the theory which relates the transport parameters to the shape of the surface rather than to some effective potential. This is especially important since modern experimental methods allow one to reconstruct the shape of the surface with a very high accuracy (see, e.g., Ref. 14).

Below we develop a very simple, versatile, and consistent method which allows to express explicitly all transport and mesoscopic parameters directly via the main characteristics of the surface, namely, via the correlation function of surface inhomogeneities. The underlying idea of our approach is to shift the difficulties from the boundary condition to the bulk equations of motions: often it is much easier to solve a complicated bulk problem with simple boundary conditions than a simpler bulk problem with complicated boundary conditions. However, in contrast to Ref. 9, we will not introduce any phenomenological bulk potentials. Instead, we will perform a canonical coordinate transformation (similar to the Migdal transformation in nuclear physics) in order to make the boundaries flat, and will look at the consequences of this transformation for transport and mesoscopic effects. Such a transformation is always possible for walls without cavities when the boundaries are described by single-valued functions.

Similar earlier ${ }^{15-18}$ for the study of diffraction patterns in the case of reflection of acoustic and/or electromagnetic waves from a rough interface. Our formalism is different, and is aimed at various transport processes along rough boundaries, including electron and phonon (photon) transport in thin films, rather than at wave patterns near the interface.

Our canonical transformation is nonlinear, and leads to a considerable complication of the bulk Hamiltonian, which acquires some additional random nonlinear terms. These terms in the bulk Hamiltonian contain all the information on initial boundary roughness. We can treat this Hamiltonian as any bulk Hamiltonian with random terms. If the amplitude of the boundary roughness is not very large, these corrections to the bulk Hamiltonian can be easily treated perturbatively. It is well known how to include perturbative corrections into the left- and righthand sides of the transport equation. The corrections to the left (dynamic) side of the transport equation are equivalent to some additional external field and are most important for the calculation of line shifts or quantized energy levels for the motion across the films. The corrections to the right-hand side (collision operator) describe the changes in the transport parameters.

Below we will concentrate mostly on the perturbative corrections to the collision operator. These perturbative terms in the collision integral can easily be translated into very transparent expressions for transport coefficients. The formalism is very simple even for rather convoluted situations.

As a result, we will be able to express all transport characteristics explicitly through the shape of the (rough) surface, i.e., the correlation function of surface inhomogeneities. Our method is applicable for inhomogeneous surfaces with the correlations of all scales, from the $\delta$ type up to macroscopic ones, such as in porous media.

Note that since we start from the exact shape of the boundary, the corresponding distortion of the bulk Hamiltonian is expressed via the shape of the surface and is very different than the effective potential used in Refs. 9, 11-13. We will see that the effective distortion is not localized near the surface, but is spread across the film. Even the operator form of this distortion is much more complicated than it was assumed before, and does not reduce to a simple potential function. What is even more important, the effective form of this distortion depends on relaxation processes in the bulk. Some general preliminary results were recently published in Ref. 19 (see also the application to transverse dynamics in spin-polarized quantum gases ${ }^{20}$ ).

Within this paper we will neglect, except for the last section, all bulk relaxation processes. Then the random boundary scattering becomes the main source of the formation of the mean free path along the walls. The absence of the bulk relaxation is the main constraint on the results that makes our method applicable mostly to thin films. In the end, we will show how this restriction can be lifted in the case of weak bulk scattering. We will also neglect all multichannel scattering effects which may be important for solid films with ideal periodic crystalline surfaces. $^{21}$ 


\section{COORDINATE TRANSFORMATION}

Let us outline the method in more detail. In principle, we can describe the transport in a semi-infinite system with one boundary, ${ }^{20}$ as well as a film with two boundaries. ${ }^{19}$ We will start from a film of the average thickness $L$ with the boundaries $x=L / 2-\xi_{1}(y, z)$ and $x=-L / 2+\xi_{2}(y, z)$. [We can also consider a $2 \mathrm{D}$ film with a rough linear boundary; then one has to disregard the variable $z$. For most of the problems in this paper, the difference between $2 \mathrm{D}$ and $3 \mathrm{D}$ films is irrelevant.] The boundary inhomogeneities are much smaller than the film thickness, $\xi_{1}, \xi_{2} \ll L$, and random so that the averages $\left\langle\xi_{1}\right\rangle=\left\langle\xi_{2}\right\rangle=0$. The results should be expressed via the binary correlation function of surface inhomogeneities. We will define the correlation functions in a somewhat simpler way than in Ref. 3:

$$
\begin{aligned}
\zeta_{i k}\left(\left|\mathbf{s}_{1}-\mathbf{s}_{2}\right|\right) & =\left\langle\xi_{i}\left(\mathbf{s}_{1}\right) \xi_{k}\left(\mathbf{s}_{2}\right)\right\rangle \\
\zeta_{i k}(\mathbf{q}) & =\int d^{2} s e^{i \mathbf{q} \cdot \mathbf{s} / \hbar} \zeta_{i k}(\mathbf{s}),
\end{aligned}
$$

where $\mathbf{s}_{1,2}(y, z)$ are coordinates along the boundaries, and $\mathbf{q}$ is the conjugate momentum along the wall. In homogeneous systems, the correlation function depends only on the distance between the points $\mathbf{s}_{1}$ and $\mathbf{s}_{2}$ and not on coordinates themselves. In most of the cases, the inhomogeneities from different boundaries are not correlated with each other, and $\varsigma_{12}=\varsigma_{21}=0$. However, this is not always the case, and we will keep the off-diagonal correlation function $\varsigma_{12}=\varsigma_{21}$. Since we neglect the bulk relaxation, most of the results below depend only on the function $\xi(\mathbf{s})=\xi_{1}(\mathbf{s})+\xi_{2}(\mathbf{s})$ and the corresponding correlation function $\zeta\left(\left|\mathbf{s}_{1}-\mathbf{s}_{2}\right|\right)=\left\langle\xi\left(\mathbf{s}_{1}\right) \xi\left(\mathbf{s}_{2}\right)\right\rangle=\zeta_{11}+\zeta_{22}+2 \zeta_{12}$.

As examples, we will consider systems with a Gaussian correlation function of the surface inhomogeneities of an average height $\ell$,

$$
\begin{aligned}
& \zeta(s)=\ell^{2} \exp \left(-s^{2} / 2 R^{2}\right), \\
& \zeta(\mathbf{q})=2 \pi \ell^{2} R^{2} \exp \left(-q^{2} R^{2} / 2 \hbar^{2}\right)
\end{aligned}
$$

and its limit for very small correlation radius $R$, i.e., the $\delta$-type correlation,

$$
\zeta(s)=\ell^{2} R^{2} \delta(s) / s, \quad \zeta(\mathbf{q})=2 \pi \ell^{2} R^{2} .
$$

If we are dealing with a $2 \mathrm{D}$ film with linear boundaries, Eqs. (2), (3) obtain the form

$$
\begin{aligned}
& \zeta(y)=\ell^{2} \exp \left(-y^{2} / 2 R^{2}\right), \\
& \zeta(q)=\sqrt{2 \pi} \ell^{2} R \exp \left(-q^{2} R^{2} / 2 \hbar^{2}\right)
\end{aligned}
$$

and

$$
\zeta(y)=\sqrt{2 \pi} \ell^{2} R \delta(y), \quad \zeta(\mathbf{q})=\sqrt{2 \pi} \ell^{2} R,
$$

respectively.

Note, that in Eqs. (2), (3) we used the simplest Gaussian parametrization for the correlation function $\zeta=$ $\zeta_{11}+\zeta_{22}+2 \zeta_{12}$, with only two characteristic parameters $\ell$ and $R$. In principle, a more consistent Gaussian parametrization should operate with three independent (and, possibly) different heights $\ell_{11}, \ell_{22}, \ell_{12}$ and correlation radii $R_{11}, R_{22}, R_{12}$ for the correlation functions $\zeta_{11}$, $\zeta_{22}, \zeta_{12}$. Though in many experimental situations the existence of extra length scales with a possible hierarchy among them may be very important, such a generalization is very straightforward, and, as we will see below, will not change the bulk of calculations, except for making the equations much more cumbersome.

The coordinate transformation

$$
X=\frac{L\left\{x-\frac{1}{2}\left[\xi_{2}(y, z)-\xi_{1}(y, z)\right]\right\}}{L-\left[\xi_{1}(y, z)+\xi_{2}(y, z)\right]}, Y=y, Z=z
$$

makes the both boundaries flat, $X=L / 2$ and $X=$ $-L / 2$. For simplicity, we consider the impenetrable reflecting walls so that the boundary condition on the walls is $\Psi=0$. Then, in new coordinates, the boundary conditions become trivial, $\Psi(L / 2)=\Psi(-L / 2)=0$.

The canonical coordinate transformation (6) should be accompanied by the conjugate transformation of momenta,

$$
p_{x}=P_{x} \frac{L}{L-\xi(y, z)}, p_{y}=P_{y}+P_{x} \frac{X \xi_{y}^{\prime}+\frac{1}{2} L\left(\xi_{2 y}^{\prime}-\xi_{1 y}^{\prime}\right)}{L-\xi_{1}(y, z)},
$$

and the same for $p_{z}\left(\xi_{1,2 y}\right.$ and $\xi_{y}^{\prime}$ are the $y$ derivatives of the functions $\xi_{1,2}$ and $\xi=\xi_{1}+\xi_{2}$ ). If the thickness of the film $L$ is large in comparison with the amplitude of surface inhomogeneities, $\xi / L \ll 1$, and the walls are relatively smooth, $\xi_{1,2 y}^{\prime}, \xi_{1,2 z}^{\prime} \ll 1$, Eqs. (6), (7) can be expanded in $\xi / L$ :

$$
\begin{aligned}
& p_{x}=P_{x}\left(1+\frac{\xi_{1}+\xi_{2}}{L}\right) \\
& p_{y}=P_{y}+P_{x}\left(\frac{1}{2}\left(\xi_{2 y}^{\prime}-\xi_{1 y}^{\prime}\right)+\frac{X\left(\xi_{1 y}^{\prime}+\xi_{2 y}^{\prime}\right)}{L}\right) .
\end{aligned}
$$

The bulk Hamiltonian $\widehat{H}$ should be expressed through the new variables. In homogeneous films, the bulk Hamiltonian depends only on momenta, $\widehat{H}=\widehat{H}_{0}(\mathbf{p})$. Then, in new variables, the Hamiltonian acquires a small "perturbation" $\widehat{V}$ :

$$
\begin{aligned}
\widehat{H} & \equiv \widehat{H}_{0}(\mathbf{p})=\widehat{H}_{0}(\mathbf{P})+\widehat{V} \\
\widehat{V} & \simeq \widehat{V}_{x} \widehat{P}_{x} \frac{\xi}{L}+\frac{1}{2}\left[\widehat{P}_{x} \widehat{\mathbf{V}}_{q} \frac{X \xi_{q}^{\prime}}{L}+\widehat{\mathbf{V}}_{q} \frac{X \xi_{q}^{\prime}}{L} \widehat{P}_{x}\right], \\
\widehat{V}_{x} & =\partial H_{0} / \partial P_{x}, \quad \widehat{\mathbf{V}}_{q}=\partial H_{0} / \partial \mathbf{Q}, \\
\xi_{q}^{\prime} & =\partial \xi / \partial \mathbf{s}, \mathbf{Q}=\left\{P_{y}, P_{z}\right\} .
\end{aligned}
$$

In general, the "perturbation" contains not only the terms (9), but also some additional terms $\widehat{V}^{\prime}$ with $\xi_{2}^{\prime}-\xi_{1}^{\prime}$ (see below). However, we will demonstrate later that these terms will disappear from the collision operator and, therefore, from the transport equation if we neglect bulk dissipation.

As a result, the problem of transport in a system with rough walls reduces to a completely equivalent prob- 
lem of transport in a system with ideal specular walls, $\Psi(L / 2)=\Psi(-L / 2)=0$, but with a perturbed bulk Hamiltonian (9). Thus the problem is shifted from the boundary condition to the bulk equations of motion. In many situations, the problem of motion of particles with random bulk Hamiltonian can be treated very easily.

\section{QUANTUM AND CLASSICAL EFFECTS IN TRANSPORT IN FILMS WITH ROUGH BOUNDARIES}

For example, if the initial Hamiltonian has the simplest quadratic form $\widehat{H}=p^{2} / 2 m$, then the same Hamiltonian in new variables obtains the "perturbation" $\widehat{V}(9)$, which, after expansion in $\xi / L$, obtains the form

$$
\begin{aligned}
\widehat{H}= & \frac{\widehat{P}^{2}}{2 m}+\widehat{V} \\
\widehat{V}= & \frac{\xi}{m L} \widehat{P}_{x}^{2}+\frac{1}{2 m}\left(X \widehat{P}_{x} \frac{\xi_{y}^{\prime}}{L} \widehat{P}_{y}\right. \\
& \left.+X \widehat{P}_{x} \frac{\xi_{z}^{\prime}}{L} \widehat{P}_{z}+\text { H.c. }\right) .
\end{aligned}
$$

Since $\langle\xi\rangle=0$, the "perturbation" $\widehat{V}$ is also random, $\langle\widehat{V}\rangle=0$.

Note, that the exact Hamiltonian contains, according to Eqs. (6), (7), not only the "perturbation" $\widehat{V}(10)$, but also the additional terms $\widehat{V^{\prime}}$ with $\xi_{1}^{\prime}-\xi_{2}^{\prime}$,

$$
\widehat{V}^{\prime}=\frac{1}{2 m}\left(\widehat{P}_{x} \frac{\xi_{1 y}^{\prime}-\xi_{2 y}^{\prime}}{L} \widehat{P}_{y}+\widehat{P}_{x} \frac{\xi_{1 z}^{\prime}-\xi_{2 z}^{\prime}}{L} \widehat{P}_{z}\right)
$$

Without bulk relaxation, when the wave functions are simple plane waves, these terms, as we will see later, will disappear from the transport equation. Therefore, we will work with the truncated "perturbation" $\widehat{V}(10)$ from the very beginning so as not to make the equations unnecessarily cumbersome. As we see, the perturbation operator $(10),(11)$ has a rather complicated and very specific form, which is different from the effective potential model. ${ }^{9}$

In thin films, the motion across the film is quantized with $P_{x}=\pi \hbar j / L$. If the thickness is very small, the distance between states with different $j$ can be so large that the transitions between these states can be effectively suppressed. Then the motion of particles along the film becomes equivalent to the $2 \mathrm{D}$ motion of particles in states $j$ in some random potentials $V^{(j)}$. In this case, the momentum across the film $P_{x}=\pi \hbar j / L$ is much larger than the momentum along the film $\mathbf{Q}=\left(P_{y}, P_{z}\right)$, and one can get the potentials $V^{(j)}$ by simply neglecting the terms with $P_{y}, P_{z}$ in Eq. (10),

$$
V^{(j)}(y, z) \simeq\left(\frac{\pi j \hbar}{L}\right)^{2} \frac{\xi(y, z)}{m L}
$$

We want to emphasize that one can use the effective potentials $V^{(j)}(y, z)(12)$ only if one neglects the transitions between the levels with different $j$. This can be done if the distance between levels is larger then the kinetic energy of motion along the film, $\pi \hbar / L \gg Q$.

If the momentum across the film is large, $P_{x}=$ $\pi \hbar j / L \gg Q$, but the distance between levels is not large and the transitions between them are allowed, one cannot simply ignore the terms with $Q$ in Eq. (10). The presence of the operator $\widehat{X}=i \hbar \partial / \partial P_{x}$ in these terms will lead to the appearance of the terms with $\delta_{P_{x}}^{\prime}\left(P_{x}-P_{x}^{\prime}\right)$ in the matrix elements. This, in turn, will result in the following integrand in the collision operator:

$$
\begin{aligned}
\delta_{P_{x}}^{\prime}\left(P_{x}-P_{x}^{\prime}\right) \delta\left(\epsilon-\epsilon^{\prime}\right)= & -\delta\left(P_{x}-P_{x}^{\prime}\right) \delta\left(\epsilon-\epsilon^{\prime}\right) \frac{\partial}{\partial P_{x}} \\
& +2 \delta\left(P_{x}-P_{x}^{\prime}\right) \delta\left(\epsilon-\epsilon^{\prime}\right) \\
& \times P_{x} \frac{\partial}{\partial\left(Q^{2}\right)}
\end{aligned}
$$

The second term in the right-hand side (rhs) of Eq. (13) makes the contribution of the terms with $Q$ in $V$ (10) to the collision integral not small in comparison with the first term in $V$, even if $P_{\boldsymbol{x}} \gg Q$. The detailed calculation in the next section shows that, under these conditions, the effective 2D potential $V_{\text {eff }}$ for the motion along the film with $P_{x}=\pi \hbar j / L \gg Q$ differs by the coefficient $\frac{1}{2}$ from the potential (12):

$$
V_{\mathrm{eff}}\left(P_{x}, y, z\right) \simeq \frac{1}{2} P_{x}^{2} \frac{\xi(y, z)}{m L} .
$$

This difference is explained by the role of transitions between the levels described by the terms with $\delta^{\prime}\left(P_{x}-P_{x}^{\prime}\right)$ (see also comments in Sec. VII).

If the levels are widely separated and we can neglect the transitions between them, we can use the potentials (12). This allows one to apply directly all available vast information on $2 \mathrm{D}$ motion in random potentials to a much less transparent problem of motion in ultrathin films with rough boundaries. Since the potential (12) is, in contrast to the "perturbation" (10), a "standard" random potential, the calculations in the ultraquantum limit are more straightforward than for classical or semiclassical problems of thicker films. In the same spirit, the ultraquantum limit for $2 \mathrm{D}$ films, i.e., very narrow strips of $2 \mathrm{D}$ films restricted by rough linear boundaries, becomes equivalent to the $1 \mathrm{D}$ motion in the $1 \mathrm{D}$ random potentials,

$$
V^{(j)}(y) \simeq\left(\frac{\pi j \hbar}{L}\right)^{2} \frac{\xi(y)}{m L}
$$

Therefore, the ultraquantum limit effectively lowers the dimensionality of the problem and simplifies the form of the random potential. These simplification will be used for the standard transport calculations in Sec. VII, and for the calculation of quantum interference and localization corrections to transport and in the discussion of the mesoscopic effects in transport in films with rough boundaries in Sec. VIII.

In classical mechanics, one can use a different ap- 
proach. Here, one can think of a particle with a bulk Hamiltonian (10) as a particle with a random coordinatedependent anisotropic effective mass,

$$
\begin{aligned}
& \left(\frac{1}{m_{\mathrm{eff}}}\right)_{x x}=\frac{1}{m}\left(1+\frac{2 \xi}{L}\right),\left(\frac{1}{m_{\mathrm{eff}}}\right)_{x y}=\frac{X \xi_{y}^{\prime}}{m L}, \\
& \left(\frac{1}{m_{\mathrm{eff}}}\right)_{x z}=\frac{X \xi_{z}^{\prime}}{m L}
\end{aligned}
$$

(the rest of the components do not change). This analogy can lead to considerable simplifications in the numerical analysis of transport in films with rough boundaries.

\section{CLASSICAL AND SEMICLASSICAL TRANSPORT OF PARTICLES WITH QUADRATIC ENERGY SPECTRUM $P^{2} / 2 M$ IN NOT VERY THIN FILMS}

In this section, we will consider not very thin films when the quantization of motion across the film is not very important. This is also an appropriate limit for a description of semi-infinite systems with a single boundary. In this classical or WKB limit, the wave functions are continuous,

$$
\Psi(\mathbf{r})=(1 / \sqrt{V}) \exp \left(i \mathbf{Q} \cdot \mathbf{s} / \hbar+i P_{\boldsymbol{x}} X / \hbar\right)
$$

where $\mathbf{Q}=\mathbf{P}_{\perp}$ is the component of momentum perpendicular to the walls, and $V$ is the total volume.

The square of the matrix element of the perturbation (10) (averaged over $\xi$ ) is determined by the correlation function (1),

$$
\begin{aligned}
\left\langle\left|V_{q P_{x}, q^{\prime} P_{x}^{\prime}}\right|^{2}\right\rangle_{\xi}= & \frac{\hbar \zeta\left(\mathbf{Q}-\mathbf{Q}^{\prime}\right)}{4 \pi L^{2} m^{2}} \\
& \times\left[2 P_{x}^{4} \delta\left(P_{x}-P_{x}^{\prime}\right)+\Omega P_{x}^{2} \delta^{\prime}\left(P_{x}-P_{x}^{\prime}\right)\right. \\
& \left.+\frac{\Omega^{2}}{8} \delta^{\prime \prime}\left(P_{x}-P_{x}^{\prime}\right)\right]
\end{aligned}
$$

where

$$
\begin{aligned}
\zeta & \equiv \zeta_{11}+\zeta_{22}+2 \zeta_{12}, \Omega\left(\mathbf{P}, \mathbf{P}^{\prime}\right) \\
& =\left(\mathbf{Q}-\mathbf{Q}^{\prime}\right) \cdot\left(P_{x} \mathbf{Q}+P_{x}^{\prime} \mathbf{Q}^{\prime}\right) .
\end{aligned}
$$

Then, the probability of transitions between states with different momenta is

$$
\begin{aligned}
W\left(\mathbf{P}, \mathbf{P}^{\prime}\right)= & \frac{2 \pi}{\hbar}\left\langle\left|V_{\mathbf{Q} P_{x}, \mathbf{Q}^{\prime} P_{x}^{\prime}}\right|^{2}\right\rangle \delta\left(\epsilon-\epsilon^{\prime}\right) \\
= & \frac{\zeta\left(\mathbf{Q}-\mathbf{Q}^{\prime}\right)}{4 \pi L^{2} m^{2}} \delta\left(\epsilon-\epsilon^{\prime}\right) \\
& \times\left[2 P_{x}^{4} \delta\left(P_{x}-P_{x}^{\prime}\right)+\Omega P_{x}^{2} \delta^{\prime}\left(P_{x}-P_{x}^{\prime}\right)\right. \\
& \left.+\frac{\Omega^{2}}{8} \delta^{\prime \prime}\left(P_{x}-P_{x}^{\prime}\right)\right] .
\end{aligned}
$$

The transport of particles in the film is described by the linearized transport equation,

$$
\partial_{t} n+\mathbf{v} \cdot \partial_{\mathbf{r}} n+\mathbf{F} \cdot \partial_{\mathbf{p}} n=L_{\mathbf{c o l l}}(n)
$$

where $n$ is the distribution function, $\mathbf{F}$ is the external force. If the only important scattering mechanism is the particle scattering by the statistically rough surface, then the effective collision integral in the rhs of the transport equation (19) is determined by transition probabilities, Eq. (18) as

$$
L_{\mathrm{coll}}=\int W\left(\mathbf{P}, \mathbf{P}^{\prime}\right)\left[n\left(1-n^{\prime}\right)-n^{\prime}(1-n)\right] \frac{d^{3} P^{\prime}}{(2 \pi \hbar)^{3}} .
$$

If we would work not only with the perturbation $\widehat{V}$ (10), but include the neglected terms $\widehat{V}^{\prime}(11)$ as well, then we would have to include into the transition probability the squares of the matrix elements $\left|V_{\mathbf{Q} P_{x}, \mathbf{Q}^{\prime} P_{x}^{\prime}}^{\prime}\right|^{2}$ and $\left(V V^{\prime}+V^{\prime} V\right)_{\mathbf{Q} P_{x}, \mathbf{Q}^{\prime} P_{x}^{\prime}}$ averaged over the surface roughness. However, since the perturbation $\widehat{V^{\prime}}(11)$ does not contain the factor $X$, these averaged squares of the matrix elements, in contrast to the averaged matrix elements $\left\langle\left|V_{\mathbf{Q} P_{x}, \mathbf{Q}^{\prime} P_{x}^{\prime}}\right|^{2}\right\rangle(17)$, will contain not the factors $\Omega\left(\mathbf{P}, \mathbf{P}^{\prime}\right) \delta^{\prime}\left(P_{x}-P_{x}^{\prime}\right)$ with the derivatives of the $\delta$ functions, but the factor $\left(Q^{2}-Q^{\prime 2}\right) \delta\left(P_{x}-P_{x}^{\prime}\right)$. As a result of the integration (20) over $d^{3} P^{\prime}$ with the additional factor $\delta\left(\epsilon-\epsilon^{\prime}\right)$ from Eq. (18), these matrix elements will vanish from the collision integral thus justifying our initial reduction of the "perturbation" to the form (10). We want to emphasize again, that the disappearance of the terms (11) can be justified only if we can use pure plane waves as unperturbed wave functions, so that the additional integrand in the collision integral has a definite $\delta$-type structure $\left(Q^{2}-Q^{\prime 2}\right) \delta\left(P_{x}-P_{x}^{\prime}\right) \delta\left(\epsilon-\epsilon^{\prime}\right)$. Otherwise, the terms (11) should be retained. These terms are important if we use different unperturbed wave functions as in the cases of noticeable bulk dissipation or large distortion of energy near the walls (see Secs. IX-XI).

Now we can turn to the explicit calculation of surface contribution to transport. We will start from mobility, or what is the same, electric conductivity. The conductivity involves the linearized transport equations $(19),(20)$ :

$$
\begin{aligned}
e \mathbf{E} \partial_{\mathbf{p}} \nu & =\int W\left(\mathbf{P}, \mathbf{P}^{\prime}\right) n_{0}\left(1-n_{0}^{\prime}\right)\left[\nu-\nu^{\prime}\right] d^{3} P^{\prime}, \\
n & =n_{0}(\epsilon)+\frac{\partial n_{0}}{\partial \varepsilon} \nu .
\end{aligned}
$$

This equation is the same as a standard equation for a conductivity problem with a scattering of electrons by bulk impurities. Therefore, we can immediately write a result at an arbitrary degree of quantum degeneracy of the electron system and arbitrary form of scattering probability $W\left(\mathbf{P}, \mathbf{P}^{\prime}\right)$ :

$$
\sigma=-\frac{4}{\pi} e^{2} L^{2} \int \frac{\partial n_{0}}{\partial \epsilon} \frac{(2 m \epsilon)^{-1 / 2}}{\zeta_{0}-\zeta_{1}} \frac{d \epsilon}{\cos ^{2} \theta} \frac{d \sin \theta}{\alpha+4 \tan ^{4} \theta}
$$


where $\theta$ is the angle between the momentum $\mathbf{P}$ and the plane of the wall,

$$
\begin{aligned}
\alpha & =\left(\eta_{0}-\eta_{1}\right) /\left(\zeta_{0}-\zeta_{1}\right), \\
\eta(P, \theta, \phi) & =\zeta(P \cos \theta, \phi)[1-\cos \phi]^{2},
\end{aligned}
$$

and $\zeta_{i}, \eta_{i}$ denote the Fourier harmonics of the functions $\zeta, \eta$ over the angle $\phi$ in the plane of the walls. As one can see, the zeroth Fourier harmonic of the correlation function of surface inhomogeneities, $\zeta(\phi)[1-\cos \phi]$, plays the role of the transport cross section for particles. If the correlation function (1) has a Gaussian form (2),

$$
\zeta\left(\mathbf{q}-\mathbf{q}^{\prime}\right)=2 \pi \ell^{2} R^{2} \exp \left(-2 q^{2} R^{2} \sin ^{2}(\phi / 2) / \hbar^{2}\right),
$$

then

$$
\begin{aligned}
\zeta_{0}-\zeta_{1} & =4 \pi^{2} \ell^{2} R^{2}{ }_{1} F_{1}\left(\frac{3}{2}, 2,-u^{2}\right) \\
\eta_{0}-\eta_{1} & =10 \pi^{2} \ell^{2} R^{2}{ }_{1} F_{1}\left(\frac{7}{2}, 4,-u^{2}\right) \\
\alpha(u) & =\frac{5}{2} \frac{{ }_{1} F_{1}\left(\frac{7}{2}, 4,-u^{2}\right)}{{ }_{1} F_{1}\left(\frac{3}{2}, 2,-u^{2}\right)}, u \equiv \sqrt{2} \frac{q R}{\hbar},
\end{aligned}
$$

where ${ }_{1} F_{1}$ is the generalized hypergeometric function,

$$
\begin{aligned}
{ }_{1} F_{1}(a, b ; x) & =\sum_{k=0}^{\infty} \frac{(a)_{k}}{(b)_{k}} \frac{x^{k}}{k !}, \\
(a)_{k} & =a(a+1)(a+2) \cdots(a+k-1),
\end{aligned}
$$

with the asymptotic behavior,

$$
\begin{aligned}
{ }_{1} F_{1}\left(\frac{3}{2}, 2,0\right)= & { }_{1} F_{1}\left(\frac{7}{2}, 4,0\right)=1, \\
u \rightarrow \infty: & { }_{1} F_{1}\left(\frac{3}{2}, 2,-u^{2}\right) \rightarrow \frac{1}{\sqrt{\pi} u^{3}}, \\
& { }_{1} F_{1}\left(\frac{7}{2}, 4,-u^{2}\right) \rightarrow \frac{6}{\sqrt{\pi} u^{7}} .
\end{aligned}
$$

The functions ${ }_{1} F_{1}\left(\frac{3}{2}, 2,-u^{2}\right)$ and ${ }_{1} F_{1}\left(\frac{7}{2}, 4,-u^{2}\right)$ are plotted in Figs. 1 and 2.

For short-range correlations with a $\delta$-type correlation function form (3), $\zeta\left(\mathbf{q}-\mathbf{q}^{\prime}\right)=2 \pi \ell^{2} R^{2}$, the argument $u$ in the above hypergeometric functions is zero, and

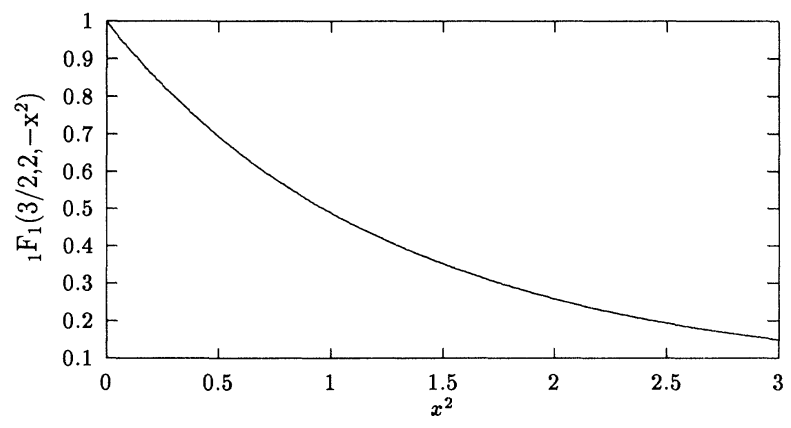

FIG. 1. Generalized hypergeometric function ${ }_{1} F_{1}\left(\frac{3}{2}, 2,-x^{2}\right)$, Eq. (25).

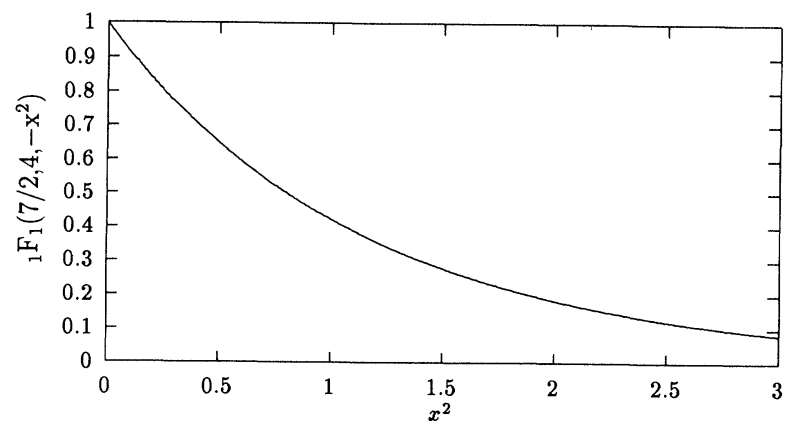

FIG. 2. Generalized hypergeometric function ${ }_{1} F_{1}\left(\frac{7}{2}, 4,-x^{2}\right)$, Eq. (25).

$\zeta_{0}-\zeta_{1}=4 \pi^{2} \ell^{2} R^{2}, \quad \eta_{0}-\eta_{1}=10 \pi^{2} \ell^{2} R^{2}, \quad \alpha(u)=5 / 2$.

In most of the calculations below we will assume that the correlations have a more general Gaussian form (2), (24), (25).

One can easily get the conductivity in the limiting cases of high temperature,

$$
\begin{aligned}
\sigma & =\frac{4 \pi^{1 / 2} e^{2} L^{2} \hbar^{3} N}{m^{2} T^{2}} \int e^{-z} \frac{z^{-1 / 2}}{\zeta_{0}-\zeta_{1}} \frac{d z}{\cos ^{2} \theta} \frac{d \sin \theta}{\alpha+4 \tan ^{4} \theta} \\
\zeta & =\zeta\left((2 m T z)^{1 / 2} \cos \theta, \phi\right),
\end{aligned}
$$

and degenerate electron systems,

$$
\begin{aligned}
\sigma & =\frac{4 e^{2} L^{2}}{\pi p_{F}} \int \frac{1}{\zeta_{0}-\zeta_{1}} \frac{1}{\cos ^{2} \theta} \frac{d \sin \theta}{\alpha+4 \tan ^{4} \theta} \\
\zeta & =\zeta\left(p_{F} \cos \theta, \phi\right)
\end{aligned}
$$

where $N$ is the bulk density of particles in the film. In the case of Gaussian correlations (2), (24), Eqs. (26) , (27) reduce to

$$
\begin{aligned}
\sigma= & \frac{32}{\pi^{3 / 2}} \frac{e^{2} L^{2} R^{2} N}{\hbar \ell^{2}} x f_{B}(x), x=\frac{\hbar}{(4 m T)^{1 / 2} R}, \\
f_{B}(x)= & x^{4} \int \frac{\exp \left[-x^{2} z^{2} / \cos ^{2} \theta\right]}{{ }_{1} F_{1}\left(\frac{3}{2}, 2,-z^{2}\right)} \frac{d z}{\cos ^{2} \theta} \\
& \times \frac{d \theta}{\alpha(z)+4 \tan ^{4} \theta},
\end{aligned}
$$

and

$$
\begin{aligned}
\sigma= & \frac{\sqrt{2} e^{2} L^{2}}{\pi^{3} \hbar \ell^{2} R} x^{2} f_{F}(x), x=\sqrt{2} \frac{p_{F} R}{\hbar} \\
f_{F}(x)= & \frac{1}{x^{3}} \int \frac{1}{{ }_{1} F_{1}\left(3 / 2,2,-x^{2} \cos ^{2} \theta\right)} \\
& \times \frac{1}{\cos ^{2} \theta} \frac{d \sin \theta}{\alpha(x \cos \theta)+4 \tan ^{4} \theta},
\end{aligned}
$$

Plots of the functions $(28),(29)$ are given in Figs. 3 and 4.

Comparison of Eqs. (28), (29) with the standard expression for the conductivity, $\sigma=e^{2} N \mathcal{L} / p$, gives the following expressions for the effective surface-induced mean 


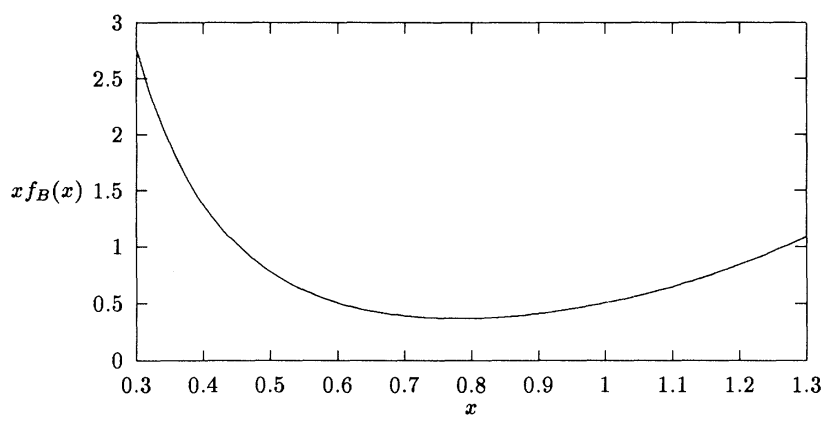

FIG. 3. Mobility of Boltzmann particles $x f_{B}(x)$, Eq. (28).

free path $\mathcal{L}$ along the film in high temperature,

$$
\mathcal{L}_{B}=\frac{16}{\pi^{3 / 2}} \frac{L^{2} R}{\ell^{2}} f_{B}(x), x=\frac{\hbar}{(4 m T)^{1 / 2} R}
$$

and degenerate

$$
\mathcal{L}_{F}=\frac{12 \sqrt{2}}{\pi} \frac{L^{2} R}{\ell^{2}} f_{F}(x)
$$

systems. Plots of functions $f_{B}(x)$ and $f_{F}(x)$ for the mean free paths $(30),(31)$ are given in Figs. 5 and 6.

The argument $x$ of the functions $f(x)$ above is the ratio of the de Broglie wavelength $\lambda$ to the correlation radius of the surface inhomogeneities $R$. In general, by the order of magnitude, the wall-induced mean free path is

$$
\mathcal{L} \sim \frac{L^{2} R}{\ell^{2}} f(R / \lambda)
$$

It is not surprising that the most effective chaotization of motion with the lowest mean free path takes place when this ratio is of the order of 1 .

The long-wave limit $R / \lambda \rightarrow 0$ corresponds to quantum reflection of particles. In this limit both functions $f_{B}$ and $f_{F}$ go to infinity, meaning that $\mathcal{L} \rightarrow \infty$. This is a simple transport consequence of the fact that for quantum reflection of long-wave particles, the reflection is effectively specular. In the opposite limiting case, $R / \lambda \rightarrow \infty$, the mean free paths also increase meaning that with widening of inhomogeneities the reflection again becomes closer

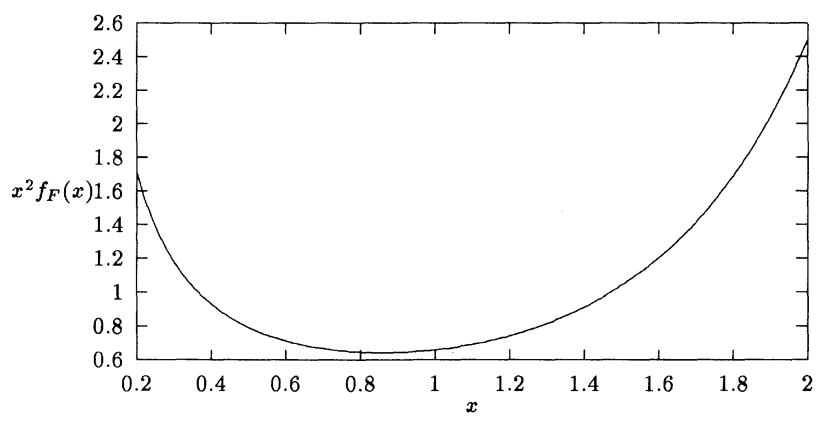

FIG. 4. Mobility of degenerate fermions $x^{2} f_{F}(x)$, Eq. (29).

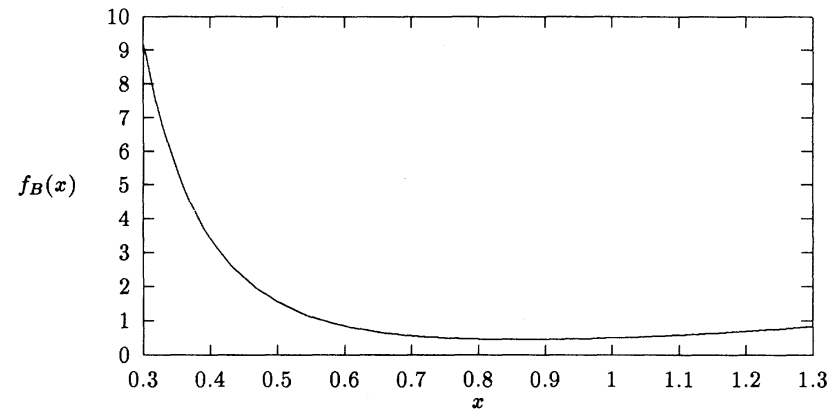

FIG. 5. Mean free path of Boltzmann particles $f_{B}(x)$, Eq. (30).

to specular.

The diffusion problem is very similar to the conductivity problem. Of course, only the diagonal components of the tensor of diffusion coefficient along the boundary, $D_{y y}$ and $D_{z z}$, are finite, while all the rest are equal to zero. These diffusion coefficients are proportional to the conductivity,

$$
D_{y y}=D_{z z}=-\frac{\pi^{2} \hbar^{3} \sigma}{2 m e^{2}} / \int \frac{\partial n_{0}}{\partial \epsilon}(2 m \epsilon)^{1 / 2} d \epsilon
$$

with the high- and low-temperature limiting cases similar to Eqs. (28),

$$
D_{y y}=D_{z z}=\frac{1}{\sqrt{\pi}} \frac{L^{2} \hbar^{3}}{\ell^{2} R^{2} m^{2} T} f_{B}\left(\frac{m T R^{2}}{\hbar^{2}}\right),
$$

and (29),

$$
D_{y y}=D_{z z}=\frac{L^{2} \hbar^{3} f_{F}\left(p_{F} R / \hbar\right)}{m \ell^{2} R^{2} p_{F}^{2}} .
$$

All other transport coefficients are calculated in the same way. What we calculated above is, in essence, the mean free path $\mathcal{L}$ along the surface imposed by scattering of particles by surface inhomogeneities. Note, that the mean free path and the transport coefficients are quadratic in the film thickness. This conclusion agrees with experimental data. ${ }^{9,22}$

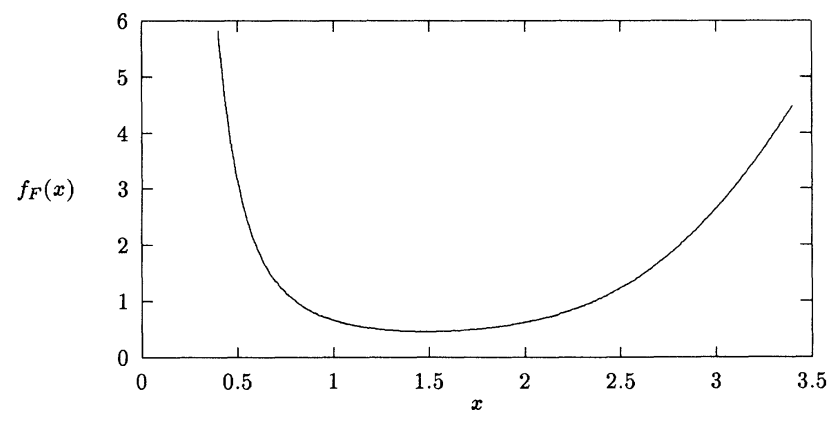

FIG. 6. Mean free path of degenerate fermions $f_{F}(x)$, Eq. (31). 


\section{SINGLE-PARTICLE DIFFUSION BETWEEN ROUGH WALLS}

In the previous section, we discussed the diffusion properties of a gas of particles contained between rough walls. A somewhat different problem is a problem of the (Brownian) motion of a single particle contained between two reflecting rough walls (a classical "bouncing ball," or a "billiard model," see, e.g., Ref. 23 and references within). Here again, the chaotization of motion is ensured by the randomness in reflection angles as a result of randomness in the wall profile. This problem is exactly equivalent to the problem of motion of particle in random bulk Hamiltonian (10) and with specular walls. The coordinate transformation (6) can simplify both the numerical and analytical analysis of the problem by reducing a complicated boundary problem to a more transparent bulk problem of the motion in the random field (10).

Of course, the Hamiltonian (10) has some distinct features (first of all, the specific momentum dependence of the random "perturbation" $\widehat{V}$ ) that make it different from the random Hamiltonians used in "typical" bulk diffusion problems. One cannot rule out the possibility that these particular features are responsible for some small deviations, of asymptotic motion of a bouncing ball from a standard diffusion behavior, which were observed in computations. However, it is still not clear whether such deviations reflect the physics of the process, or are the features of the computational models involved. [The computational analysis of the motion of the bouncing ball on the basis of the transformation (6) might, in principle, give an independent insight into this problem.] We will not go into a discussion of this problem here, and will simply assume that the motion of the bouncing ball has a diffusive character. What is more, we will postpone the study of all quantum and classical interference effects, ${ }^{23,24}$ localization anomalies, etc., until Sec. VIII, and will restrict ourselves in this section to the study of classical diffusion exclusively.

The appropriate transport equation for a singleparticle diffusion is the Focker-Plank equation,

$$
\partial_{t} n=\partial_{\mathbf{p}}\left[\mathbf{G} n+\partial_{\mathbf{p}} \overleftrightarrow{D^{(p)}} n\right]
$$

rather than the Boltzmann equation (19). Here, the tensor of diffusion coefficients in momentum space is equal to

$$
D_{i k}^{(p)}=\frac{1}{2} \int Q_{i} Q_{k} W\left(\mathbf{P}, \mathbf{P}^{\prime}\right) d^{3} Q, \quad \mathbf{Q}=\mathbf{P}-\mathbf{P}^{\prime}
$$

while the vector $\mathbf{G}$ is

$$
\mathbf{G}=\int \mathbf{Q} W\left(\mathbf{P}, \mathbf{P}^{\prime}\right) d^{3} Q
$$

and we can still use Eq. (18) for the transition probability $W\left(\mathbf{P}, \mathbf{P}^{\prime}\right)$.

The single-particle diffusion coefficient in momentum

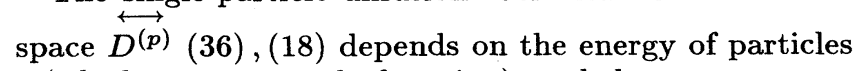
$\epsilon$ (which is an integral of motion), and the average component of the momentum perpendicular to the walls, $P_{\boldsymbol{x}}$.
This coefficient can easily be calculated. However, we are interested not in $\overleftrightarrow{D^{(p)}}$, but in the diffusion coefficients $D_{y y}=D_{z z}$ in real space. The coefficients are determined by the characteristic diffusion time, which, in turn, is determined by $\stackrel{\leftrightarrow}{D^{(p)}}$ as the time in which the components of momentum along the walls change from $\mathbf{q}$ to $-\mathbf{q}$ :

$$
D_{y y}=D_{z z}=\frac{2 \pi \hbar^{3} L^{2}}{\zeta_{0}-\zeta_{1}} \frac{\epsilon-P_{x}^{2} / 2 m}{\alpha\left(2 m \epsilon-P_{x}^{2}\right)^{2} / 4+P_{x}^{4}} .
$$

It is not at all surprising that Eq. (37) is similar to the integrand in Eqs. (22), (32). In the case of Gaussian correlations, the single-particle diffusion coefficient is

$$
\begin{aligned}
& D_{y y}=D_{z z}= \frac{\hbar^{3} L^{2}}{2 \pi \ell^{2} R^{2}} \frac{1}{{ }_{1} F_{1}\left(3 / 2,2,-u^{2}\right)} \\
& \times \frac{\epsilon-P_{x}^{2} / 2 m}{\alpha\left(2 m \epsilon-P_{x}^{2}\right)^{2} / 4+P_{x}^{4}}, \\
& u^{2}=\frac{2 R^{2}}{\hbar^{2}}\left(2 m \epsilon-P_{x}^{2}\right) .
\end{aligned}
$$

Some numerical simulations imply that the asymptotic motion of a bouncing ball and a particle in a random 2D potential have nondiffusion anomalies. At present, it is not clear whether these observations reflect some general physics laws, or are some properties of computational algorithms. However, our method indicates that if these asymptotic anomalies are real, such anomalies for $2 \mathrm{D}$ diffusion in random potential and for a bouncing ball model should be strongly related to each other.

\section{TRANSPORT OF PARTICLES WITH AN ARBITRARY ENERGY SPECTRUM $\epsilon(P)$. APPLICATIONS TO PHONONS AND PHOTONS, $\epsilon=C P$}

The above results can be easily modified so as to describe the diffusion of particles with arbitrary energy spectra $\epsilon(\mathbf{p})$. Some of the applications of these results deal with solid-state quasiparticles. Below we will perform the calculations in the semiclassical approximation, which will ignore both the interband transitions and the change of crystal symmetry at the surface responsible for the multichannel reflection. ${ }^{21}$

The unperturbed wave functions for particles with an arbitrary spectrum $\epsilon(\mathbf{p})$ are still plane waves, and the transition probability $W\left(\mathbf{P}, \mathbf{P}^{\prime}\right)$ for the "perturbation" (10) is similar to (18):

$$
\begin{aligned}
W\left(\mathbf{P}, \mathbf{P}^{\prime}\right)= & \frac{\zeta\left(\mathbf{q}-\mathbf{q}^{\prime}\right)}{4 \pi L^{2}} \delta\left(\epsilon^{\prime}-\epsilon\right) \\
& \times\left[2 V_{x}^{2} P_{x}^{2} \delta\left(P_{x}-P_{x}^{\prime}\right)+\Omega V_{x} P_{x} \delta^{\prime}\left(P_{x}-P_{x}^{\prime}\right)\right. \\
& \left.+\frac{\Omega^{2}}{8} \delta^{\prime \prime}\left(P_{x}-P_{x}^{\prime}\right)\right] \\
\Omega\left(\mathbf{P}, \mathbf{P}^{\prime}\right)= & \left(P_{x} \mathbf{V}_{\mathbf{q}}-P_{x}^{\prime} \mathbf{V}_{\mathbf{q}^{\prime}}^{\prime}\right) \cdot\left(\mathbf{Q}-\mathbf{Q}^{\prime}\right)
\end{aligned}
$$

For the sake of simplicity, let us consider the spec- 
tra which are isotropic in the plane parallel to the walls, $\epsilon(\mathbf{p})=\epsilon\left(q, p_{x}\right)$, i.e., do not depend on the direction of the vector $\mathbf{q}$. Then the collision integral is practically the same as in Eq. (20), while the left-hand side (lhs) of the transport equation differs from Eq. (18) only by the form of the velocity $\mathbf{v}=\partial \epsilon / \partial \mathbf{p}$. As a result, the diffusion coefficient for particles with arbitrary spectrum obtains the form Eq. (32),

$$
\begin{aligned}
D_{y y}=D_{z z}= & 16 \pi L^{2} \hbar^{3}\left[\int \frac{\partial n_{0}}{\partial \epsilon} Q d Q d P_{x}\right]^{-1} \int \frac{\partial n_{0}}{\partial \epsilon} \\
& \times \frac{V_{q}^{2} Q d Q d P_{x}}{V_{q} Q^{3}\left(\eta_{0}-\eta_{1}\right)+4 Q V_{x}^{2} P_{x}^{2}\left(\varsigma_{0}-\varsigma_{1}\right) / V_{q}},
\end{aligned}
$$

where $V_{q}=\partial \epsilon / \partial Q$ and $V_{x}$ are the components of velocity. In the case of Gaussian correlations of surface inhomogeneities, this equation reduces to

$$
\begin{aligned}
D_{y y}=D_{z z}= & \frac{4 L^{2} \hbar^{3}}{\pi \ell^{2} R^{2}}\left[\int \frac{\partial n_{0}}{\partial \epsilon} Q d Q d P_{x}\right]^{-1} \\
& \times \int \frac{\partial n_{0}}{\partial \epsilon} \frac{1}{{ }_{1} F_{1}\left(3 / 2,2,-2 Q^{2} R^{2} / \hbar^{2}\right)} \\
& \times \frac{V_{q}^{2} Q d Q d P_{x}}{\alpha V_{q} Q^{3}+4 Q V_{x}^{2} P_{x}^{2} / V_{q}} .
\end{aligned}
$$

As an example, let us describe classical diffusion of "relativistic" particles with linear spectrum $\epsilon=c p$, such as photons or phonons. In this case, the perturbation (9) has the form

$$
\delta \widehat{H}=c(m / 2)^{1 / 2} \widehat{H}_{0}^{-1 / 2} \widehat{V},
$$

where $\widehat{H}_{0}=p^{2} / 2 m$, and the operator $\widehat{V}$ is given by Eq. (10). The matrix elements of this perturbation are

$$
\left\langle\mathbf{p}^{\prime}\left|\delta \widehat{H}_{1}\right| \mathbf{p}\right\rangle=\frac{c m}{\left(P_{x}^{2}+P_{y}^{2}+P_{z}^{2}\right)^{1 / 2}} V_{\mathbf{p}^{\prime}},
$$

with $V_{\mathbf{p p}}(16)$. Then the scattering probability differs from Eq. (18) only by the factor $c^{2} m^{2} / P^{2}$, and the singleparticle diffusion coefficient is very similar to Eqs. (37) and (38),

$$
D_{y y}=D_{z z}=\frac{\pi \hbar^{3} c^{2} L^{2}}{\epsilon\left(\zeta_{0}-\zeta_{1}\right)} \frac{\epsilon^{2} / c^{2}-P_{x}^{2}}{\alpha\left(\epsilon^{2} / c^{2}-P_{x}^{2}\right)^{2} / 4+P_{x}^{4}}
$$

or, in the Gaussian case (25),

$$
\begin{aligned}
D_{y y}=D_{z z}= & \frac{\hbar^{3} c^{2} L^{2}}{\pi \epsilon \ell^{2} R^{2}} \frac{1}{{ }_{1} F_{1}\left(3 / 2,2,-u^{2}\right)} \\
& \times \frac{\epsilon^{2} / c^{2}-P_{x}^{2}}{\alpha\left(\epsilon^{2} / c^{2}-P_{x}^{2}\right)^{2}+4 P_{x}^{4}}, \\
u^{2}= & 2 \frac{R^{2}}{\hbar^{2}}\left(\frac{\epsilon^{2}}{c^{2}}-P_{x}^{2}\right) .
\end{aligned}
$$

In principle, one can also calculate the diffusion coefficient for anisotropic bulk spectra $\epsilon(\mathbf{p})$. In this case, the transport equation becomes a cumbersome matrix equation in angular harmonics, and the results are much less transparent though the derivation is rather straightforward.

\section{DIFFUSION OF PARTICLES WITH DISCRETE QUANTIZED STATES FOR THE MOTION ACROSS THE FILM}

In many cases, including very thin films, it is impossible to neglect quantization of motion across the film. In our geometry, this does not change the dependence of the wave functions on spatial coordinates along the film which remains $\exp (i \mathbf{q} \cdot \mathbf{r})$, and the quantization affects only the dependence on coordinate $x$ across the film. The wave functions in new variables are

$$
\Psi_{j}(\mathbf{r})=\sqrt{2 / V} \exp (i \mathbf{q} \cdot \mathbf{s}) \sin (\pi j X / L) .
$$

The transition to classical and WKB cases of Sec. IV with wave functions (12) corresponds to large wave numbers $j$ when the last factor should be substituted by $\exp \left(i P_{x} X\right) / \sqrt{2}$.

The matrix elements of the perturbation (10) with the wave functions (45) are rather cumbersome,

$$
\begin{aligned}
V_{\mathbf{q} j, \mathbf{q}^{\prime} j^{\prime}}= & V_{\mathbf{q} j, \mathbf{q}^{\prime} j^{\prime}}^{(x)}+V_{\mathbf{q} j, \mathbf{q}^{\prime} j^{\prime}}^{(y)}+V_{\mathbf{q} j, \mathbf{q}^{\prime} j^{\prime}}^{(z)}, \\
V_{\mathbf{q} j, \mathbf{q}^{\prime} j^{\prime}}^{(x)}= & \frac{\delta_{j j^{\prime}}}{m L} \frac{\pi^{2}\left(j^{2}+j^{\prime 2}\right)}{L^{2}} \xi\left(\mathbf{q}-\mathbf{q}^{\prime}\right), \\
V_{\mathbf{q} j, \mathbf{q}^{\prime} j^{\prime}}^{(y)}= & \frac{(-1)^{j+j^{\prime}}}{2 m L} \xi\left(\mathbf{q}-\mathbf{q}^{\prime}\right)\left(Q_{y}^{\prime}-Q_{y}\right) \\
& \times\left[Q_{y}^{\prime} j^{\prime}\left(\frac{1}{j+j^{\prime}}+\frac{1}{j-j^{\prime}}\left(1-\delta_{j j^{\prime}}\right)\right)\right. \\
& \left.-Q_{y} j\left(\frac{1}{j+j^{\prime}}-\frac{1}{j-j^{\prime}}\left(1-\delta_{j j^{\prime}}\right)\right)\right],
\end{aligned}
$$

and the collision integral (20) becomes a matrix in the quantum numbers $j$ :

$$
\begin{aligned}
L_{j}= & \frac{1}{2 \pi \hbar^{3} m^{2} L^{2}} \int d^{2} Q^{\prime} \zeta\left(\mathbf{Q}-\mathbf{Q}^{\prime}\right) \sum_{j^{\prime}}\left[n_{j^{\prime}}\left(\mathbf{Q}^{\prime}\right)\right. \\
& \left.-n_{j}(\mathbf{Q})\right] \delta\left(\epsilon_{j^{\prime} \mathbf{Q}^{\prime}}-\epsilon_{j \mathbf{Q}}\right) \\
& \times\left\{\delta_{j j^{\prime}}\left[\frac{1}{4}\left(\mathbf{Q}-\mathbf{Q}^{\prime}\right)^{2}+\left(\frac{\pi \hbar j}{L}\right)^{2}\right]^{2}\right. \\
& \left.+\frac{\left(1-\delta_{j j^{\prime}}\right) j^{2} j^{\prime 2}}{\left(j^{2}-j^{\prime 2}\right)^{2}}\left(Q^{\prime 2}-Q^{2}\right)^{2}\right\}
\end{aligned}
$$

where $\epsilon_{j \mathbf{Q}}=\left[Q^{2}+(\pi j \hbar / L)^{2}\right] / 2 m$.

In principle, surface inhomogeneities lead to three effects, namely, to the change of quantized energy levels $\epsilon_{j \mathbf{Q}}$, interstate transitions $j \rightarrow j^{\prime}$ with diffusion between energy levels, and diffusion of particles on the same level. In this paper, we ignore the first effect completely and do not consider the corrections to the dynamic, lhs, of the transport equation. (The effect of shape-induced changes in eigenstates on transport is discussed in some detail in Ref. 25 for inhomogeneous quantum wires of nonrandom shapes. Our approach to transport in channels of complex shapes can be used as a basis for an alternative numerical algorithm applicable for wires of random as well 
as nonrandom shapes.) The second and the third effects are intermixed and are described by the matrix transport equation with the interstate transitions ensured by the off-diagonal elements $\left(1-\delta_{j j^{\prime}}\right)$ of the collision integral (47).

If the distance between energy levels is large (thin films) and we are interested only in diffusion on the lowest levels, the off-diagonal elements of the collision integral (47) are small, and the coupling between the states with different $j$ is negligible. Then the main effect of surface roughness is the diffusion of particles without interlevel transitions. This process is described by the transport equation,

$$
\partial_{t} n\left(\epsilon_{j}, \mathbf{q}\right)+\frac{\mathbf{q}}{m} \partial_{\mathbf{r}} n\left(\epsilon_{j}, \mathbf{q}\right)+\mathbf{F} \partial_{\mathbf{q}} n\left(\epsilon_{j}, \mathbf{q}\right)=L_{j}\left\{n_{j}\right\}
$$

with only diagonal elements $\delta_{j j^{\prime}}$ in the collision integral (47). Then the collision integral (47) obtains exactly the same form as when we would use for the perturbation $\widehat{V}$, the form (12)

$$
V_{j}(y, z)=\left(\frac{\pi j \hbar}{L}\right)^{2} \frac{\xi(y, z)}{m L},
$$

from the very beginning. If the interlevel transitions are allowed and the off-diagonal terms cannot be neglected, the collision integral (47) provides the discrete version of the collision integral in Sec. IV. The off-diagonal terms serve as analogs of the terms with $\delta^{\prime}\left(P_{x}-P_{x}^{\prime}\right)$, and, if the interlevel transitions are possible, will lead to an important renormalization of the effective potential for $2 \mathrm{D}$ motion along the film even if $P_{x} \gg Q$.

The potentials (10) are just simple random $2 \mathrm{D}$ potentials without any surface-induced peculiarities of the general expression (10). If we assume that the $2 \mathrm{D}$ motion in random potential is diffusive (there are some numerical indications that this is not always the case; however, this issue is still controversial), then the problem becomes "trivial," or, at least, standard.

The diagonal elements in the collision integral (47) corresponding to the bulk perturbation (12) have a very simple form,

$$
\begin{aligned}
L_{j}\left\{n_{j}\right\}= & \frac{4}{\pi \hbar^{3} m L^{2}}\left(\frac{\pi \hbar j}{L}\right)^{4} \int d^{2} Q^{\prime} \zeta\left(\mathbf{Q}-\mathbf{Q}^{\prime}\right) \\
& \times\left[n_{j}\left(\mathbf{Q}^{\prime}\right)-n_{j}(\mathbf{Q})\right] \delta\left(Q^{\prime 2}-Q^{2}\right) .
\end{aligned}
$$

The transport equation (48) with the collision integral (49) is a standard $2 \mathrm{D}$ transport problem not different from any other problem with weak impurity scattering. This problem can be easily solved in the same way as Eq. (19) and (20) in Sec. IV. The final expression for the conductivity $\sigma^{(j)}$ for the single-level motion trivially depends on the level number $j$, and is equal to

$$
\sigma_{x x}^{(j)}=\sigma_{y y}^{(j)}=-\frac{e^{2} L^{2} \hbar}{8 m}\left(\frac{L}{\pi \hbar j}\right)^{4} \int \frac{\partial n_{0}}{\partial \epsilon} \frac{Q^{3} d Q}{\zeta_{0}-\zeta_{1}} .
$$

In the cases of Gaussian and $\delta$ correlations (2) and (3), this expression reduces to

$$
\begin{aligned}
\sigma_{x x}^{(j)}=\sigma_{y y}^{(j)}=- & \frac{e^{2} L^{2} \hbar}{32 m \pi^{2} \ell^{2} R^{2}}\left(\frac{L}{\pi \hbar j}\right)^{4} \\
& \times \int \frac{\partial n_{0}}{\partial \epsilon} \frac{Q^{3} d Q}{{ }_{1} F_{1}\left(\frac{3}{2}, 2,-2 Q^{2} R^{2} / \hbar^{2}\right)}
\end{aligned}
$$

and

$$
\sigma_{x x}^{(j)}=\sigma_{y y}^{(j)}=-\frac{e^{2} L^{2} \hbar}{32 m \pi^{2} \ell^{2} R^{2}}\left(\frac{L}{\pi \hbar j}\right)^{4} \int \frac{\partial n_{0}}{\partial \epsilon} Q^{3} d Q .
$$

In the Boltzmann and degenerate limits, Eq. (51) reduces to [cf. Eqs. (28), (29)]

$$
\begin{aligned}
\sigma_{x x}^{(j)} & =\sigma_{y y}^{(j)}=\frac{e^{2} L^{2} \hbar^{3}}{4 \pi \ell^{2} R^{2}}\left(\frac{L}{\pi \hbar j}\right)^{4} x f_{B}^{(j)}(x), \\
x & =\frac{\hbar}{2(m T)^{1 / 2}}, \\
f_{B}^{(j)}(x) & =x^{3} \int \frac{z^{3} \exp \left(-z^{2} x^{2}\right) d z}{{ }_{1} F_{1}\left(\frac{3}{2}, 2,-z^{2}\right)}
\end{aligned}
$$

and

$$
\begin{aligned}
\sigma_{x x}^{(j)} & =\sigma_{y y}^{(j)}=\frac{e^{2} L^{2} \hbar^{3} N^{(j)}}{16 \pi \ell^{2} R^{2}}\left(\frac{L}{\pi \hbar j}\right)^{4} \frac{1}{x} f_{F}^{(j)}(x), \\
x & =\left(4 \pi N^{(j)} R^{2}\right)^{1 / 2}, \\
f_{F}^{(j)}(x) & =\frac{x}{{ }_{1} F_{1}\left(\frac{3}{2}, 2,-x^{2}\right)},
\end{aligned}
$$

respectively $\left(N^{(j)}\right.$ is the 2D density of particles on the level $j$ ). Functions $f_{B, F}^{(j)}(52),(53)$ are plotted in Figs. 7 and 8 . The expressions for the corresponding diffusion coefficients are similar to (32),

$$
D_{y y}^{(j)}=D_{z z}^{(j)}=-\frac{\pi^{2} \hbar^{3}}{2 m e^{2}} \sigma^{(j)} / \int \frac{\partial n_{0}}{\partial \epsilon}(2 m \epsilon)^{1 / 2} d \epsilon
$$

while the effective mean free path

$$
\begin{aligned}
\mathcal{L}^{(j)} & =\frac{\bar{Q}^{(j)} \sigma^{(j)}}{e^{2} N^{(j)}} \\
\mathcal{L}_{B}^{(j)} & =\frac{L^{2}}{8 \pi \ell^{2} R^{3}}\left(\frac{L}{\pi j}\right)^{4} f_{B}^{(j)}(x), \\
\mathcal{L}_{F}^{(j)} & =\frac{\sqrt{2} L^{2}}{32 \pi \ell^{2} R^{3}}\left(\frac{L}{\pi j}\right)^{4} f_{F}^{(j)}(x),
\end{aligned}
$$

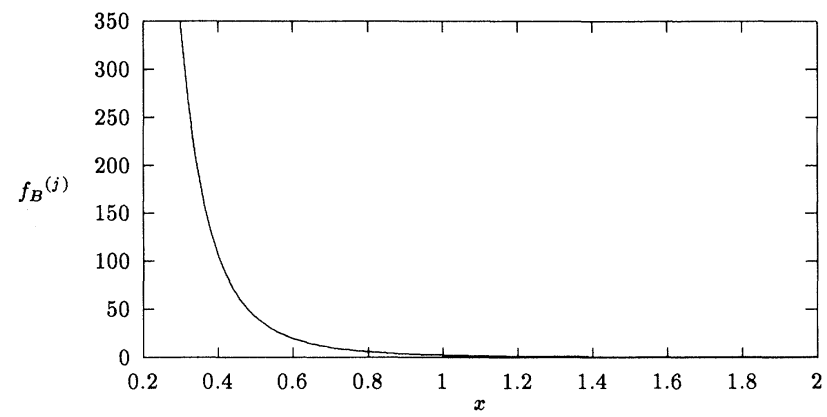

FIG. 7. Function $f_{B}^{(j)}(x)$, Eq. (52) 


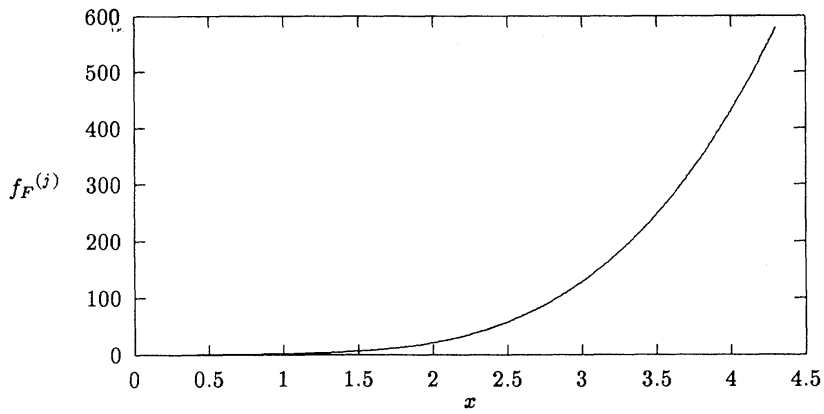

FIG. 8. Function $f_{F}^{(j)}(x)$, Eq. (53) .

where $\bar{Q}^{(j)}$ is the average (thermal) momentum along the film for the motion on the level $j$.

Of course, the $2 \mathrm{D}$ motion in weak random potential (12) results in localization of particles along the film with large localization radius (see Sec. VIII).

\section{INTERFERENCE CORRECTIONS TO CONDUCTIVITY, LOCALIZATION, AND MESOSCOPIC EFFECTS}

As it was mentioned before, the collisions with rough walls limit the mean free path along the film. Another effect of randomness of the wall scattering is the appearance of quantum interference effects similar to those caused by scattering by usual random bulk imperfections (impurities) ${ }^{26,27}$ These interference effects manifest themselves in, for example, quantum corrections to conductivity and different mesoscopic effects. Though it is already known that random boundary scattering leads to different interference and localization effects (see, e.g., Refs. 9 and 24 and references therein), our approach allows us to recover known results and additional ones in a very simple and straightforward way. The reason is that practically the only information, necessary for calculation of such quantum and interference corrections, is the mean free path and diffusion coefficient. Thus, we will be able to get simple expressions relating the mesoscopic length scales to the parameters of the correlation function of surface inhomogeneities. As we will see, the interference corrections strongly depend on whether the motion of particles across the film is classical (thick films) or quantized with large separation between levels (very thin films).

\section{A. Interference corrections to conductivity in thick $3 \mathrm{D}$ films}

The simplest case is the classical motion of particles in relatively thick $3 \mathrm{D}$ films with $2 \mathrm{D}$ rough walls. The coordinate transformation makes these films equivalent to the films with flat walls and random bulk imperfections (10). The quantum corrections to conductivity of $3 \mathrm{D}$ films with random bulk imperfections are given by a standard expression,

$$
\frac{\Delta \sigma}{\sigma}=-\int_{\tau}^{\tau_{\phi}} v \lambda^{2}\left(D_{y y} t\right)^{-3 / 2} d t
$$

where $\tau$ and $\tau_{\phi}$ are the momentum and energy relaxation times, $\lambda$ is the de Broglie wavelength of particles, and the tensor of diffusion coefficients $D_{i k}$ is calculated in previous sections. The calculations are especially simple in the usual limit $\tau_{\phi} \rightarrow \infty$. Fortunately, this limit corresponds to our initial assumption that the bulk relaxation processes (scattering on impurities, particle-particle collisions, etc.) are negligible. In this limit,

$$
\frac{\Delta \sigma}{\sigma}=-\frac{8 \pi^{2} \hbar^{2}}{m^{2} D_{y y}^{2}},
$$

with $D_{y y}$ given by Eqs. (30) - (34).

\section{B. 3D films with strong quantization of motion across the film}

The situation becomes different if the motion of particles across the films is distinctly quantized with large separation between the energy levels. This situation occurs for very thin films with $P_{x}=\pi j \hbar / L$. If the thickness $L$ is so small that $P_{x} \gg P_{y, z}$, and the distance between levels is so large that the transitions between them are effectively suppressed, then the motion of particles on each level becomes effectively a $2 \mathrm{D}$ motion in a $2 \mathrm{D}$ random potential (12)

$$
V^{(j)}(y, z)=\left(\frac{\pi j \hbar}{L}\right)^{2} \frac{\xi(y, z)}{m L}
$$

(see also Sec. VII). This random potential is not different from any other random potentials studied in the localization theory, and we can use directly a wide variety of standard results ${ }^{26,27}$ practically without any modifications or additional calculations. Of course, the only important parameter is the mean free path along the film calculated in Secs. IV and VII. For example, the localization length $\mathcal{R}$ for particles in very thin film with rough walls is exponentially large and is given by a standard expression for the $2 \mathrm{D}$ motion in a weak random potential:

$$
\mathcal{R}^{(j)} \sim \mathcal{L}^{(j)} \exp \left(\pi^{2} \mathcal{L}^{(j)} / \lambda\right)
$$

where $\lambda$ is the de Broglie wavelength of particles, and the effective mean free path of particles on the quantized level $j, \mathcal{L}^{(j)}$, is expressed via parameters of the correlation function of surface inhomogeneities as (54), (30), (31),

$$
\mathcal{L}^{(j)} \sim \frac{L^{2} \hbar^{4}}{\ell^{2} R^{3}}\left(\frac{L}{\pi \hbar j}\right)^{4} f^{(j)}(x) .
$$

\section{2D films with strong quantization of motion across the film}

The above result describes the effectively $2 \mathrm{D}$ character of motion of particles in very thin $3 \mathrm{D}$ films. The situation 
in 2D films (narrow strips of 2D films restricted by random linear boundaries) is different. Here, in the case of highly quantized motion across the strip, the motion on each level becomes effectively $1 \mathrm{D}$ in the random potential (15),

$$
V_{j}(y)=\left(\frac{\pi j \hbar}{L}\right)^{2} \frac{\xi(y)}{m L}
$$

(as above, we assume that the film is so narrow that the quantized momentum across the film, $P_{x}=2 \pi j \hbar / L$, is much larger than the momentum along the film, $P_{y}$, and that the transitions between levels are suppressed).

The localization implications of the $1 \mathrm{D}$ motion in the weak random potential (15) are obvious. What is more, we can give an explicit expression for the density of states assuming the $\delta$ correlation of boundary inhomogeneities, Eq. (5),

$$
\left\langle\xi\left(y_{1}\right) \xi\left(y_{2}\right)\right\rangle=\sqrt{2 \pi} \ell^{2} R \delta\left(y_{1}-y_{2}\right) .
$$

Under these conditions, the correlation function for the "perturbation" (15) is

$$
\left\langle V_{j}\left(y_{1}\right) V_{j}\left(y_{2}\right)\right\rangle=\sqrt{2 \pi} \frac{(\pi j \hbar)^{4} \ell^{2} R}{m^{2} L^{6}} \delta\left(y_{1}-y_{2}\right)
$$

and we can neglect the off-diagonal matrix elements of the perturbation responsible for the transitions between the states with different quantum numbers $j$. [Note, that the correlation function in Ref. 19 differs from Eq. (59) by a trivial coefficient.] Then we can use the wellknown result ${ }^{28}$ for the particle density of states in the $\delta$-correlated potential (59):

$$
\begin{aligned}
\nu^{-1}(E) & =\frac{2 \pi^{2}\left(j^{4} \ell^{2} R / 16\right)^{1 / 3}}{L^{2}} \int_{0}^{\infty} \frac{d z}{\sqrt{z}} \exp \left(-\frac{z^{3}}{24}-z \mathcal{E}\right), \\
\mathcal{E} & =\frac{E m L^{2}}{(2 \pi)^{3} \hbar^{2}}\left(\frac{16 L^{3}}{j^{4} \ell^{2} R}\right)^{2 / 3}
\end{aligned}
$$

This result describes the localization of the particle in a narrow $2 \mathrm{D}$ film with rough boundaries.

\section{EFFECT OF ENERGY DISTORTION NEAR THE BOUNDARIES ON TRANSPORT}

In this section, we will consider how the changes in potential energy near the surface can affect the transport in films with rough boundaries. Let us suppose, that the particle potential near the walls is somehow distorted, and that this distortion $\delta U$ depends only on the distance from the wall,

$$
\begin{aligned}
\delta U(x, y, z)= & U\left[x-L / 2+\xi_{1}(y, z)\right] \\
& +U\left[x+L / 2-\xi_{2}(y, z)\right] .
\end{aligned}
$$

After the coordinate transformation, this potential can be split into regular,

$$
U_{\text {reg }}(X)=U(X-L / 2)+U(X+L / 2),
$$

and random,

$$
\begin{aligned}
U_{\mathrm{ir}}(X, Y, Z)= & \frac{\partial U(X-L / 2)}{\partial X} \xi_{1}(Y, Z) \\
& -\frac{\partial U(X+L / 2)}{\partial X} \xi_{2}(Y, Z),
\end{aligned}
$$

parts.

The role of these two contributions to the potential is different. The random part $(63), U_{\mathrm{ir}}$, should be considered in the same way as and simultaneously with the perturbation $\widehat{V}(10)$. Together these two random functions form an effective perturbation $\widehat{V}_{\text {eff }}=\widehat{V}+U_{\text {ir }}$, which should be used everywhere instead of $\widehat{V}$. In the calculations of Sec. IV, this amounts only to a trivial change in collision integral (20). If one neglects the effect of the regular part (10), the effect of substitution of $\widehat{V}$ by $\widehat{V}_{\text {eff }}$ on transport reduces to the change in the Fourier components of the correlation function $\zeta$ in the denominators of the integrands for the transport coefficients in Sec. IV:

$$
\zeta_{i} \rightarrow \zeta_{\mathrm{eff} i}, \alpha \rightarrow \alpha_{\mathrm{eff}}
$$

Depending on the energy distortion, the random distortion correction (63) can dominate over or be much smaller than the contribution of the "bare" perturbation (10).

The presence of the regular part $(62), U_{\text {reg }}(X)$, somewhat complicates the situation. This term should be treated differently depending on whether the situation is classical (and WKB) or quantum. In a classical case, the potential $U_{\text {reg }}(X)$ modifies to the force term in the lhs of the transport equation (19). This changes the equilibrium distribution function, which obtains the coordinate dependence, $n_{0}\left[P^{2} / 2 m+U_{\text {reg }}(X)\right]$. Since we are interested in mobility and diffusion in the directions $y, z$ along the film, the main effect of this change is that the momentum integrations in Eqs. (22), (28), (29) should be supplemented by the spatial integration over $d X / L$ (of course, one should also substitute $\zeta$ by $\zeta_{\text {eff }}$ so that to include the effect of $\left.U_{\text {ir }}\right)$. Note, that above we always neglected all the corrections to the lhs of the transport equation from the perturbation (10), including, for example, the averaged second order perturbation correction to the Hamiltonian.

A more important change is the distortion of the wave functions used for calculation of the matrix elements. As was explained in Sec. IV, the change in the wave functions should automatically invoke the use of extra terms (11) in the perturbation $\widehat{V}$. One of the most important results will be that correlation function of the surface inhomogeneities will enter the result not as $\varsigma=\varsigma_{11}+\varsigma_{22}+2 \varsigma_{12}$, but as a more complicated combination of the correlation functions $\varsigma_{i k}$ (in particular, there should be a relative decrease in the role cross correlations $\varsigma_{12}$, with an increase in potential distortions near the walls).

In the quantum case with discrete energy states for motion across the film, the main change occurs in the calculation of the matrix elements of the perturbation. Now the matrix elements of the perturbation $\widehat{V}_{\text {eff }}$ should 
be calculated not with the help of the wave functions (12) or (45), but the functions

$$
\Psi_{j}(\mathbf{r})=\sqrt{2 / V} \exp (i \mathbf{q} \cdot \mathbf{s}) \widetilde{\Psi}_{j},
$$

where $\widetilde{\Psi}_{j}$ are the eigenfunctions for the $1 \mathrm{D}$ motion of particle between the walls $X= \pm L / 2$ in the potential $U_{\text {reg }}(X)(62)$ with the eigenvalues $\epsilon_{j}$. Simultaneously, one should include into the perturbation all the terms (11). After the matrix elements are recalculated, the solution of the transport equation is similar to that in Sec. VII.

\section{EFFECT OF BULK IMPURITIES}

We can also take into account the effect of scattering of particles by impurities, and the interference between boundary and impurity scattering. This is especially simple when the impurity scattering is weak and can be described by the collision integral in the Born approximation.

Scattering by impurities is described by the addition of the random particle-impurity interaction potential $U(\mathbf{r})$ to the bulk Hamiltonian. Without scattering by surface inhomogeneities, this potential would lead to the following collision integral in the transport equation [cf. Eq. (21)]:

$$
\frac{2 \pi}{\hbar} \int\left\langle\left|U_{\mathbf{p} \mathbf{p}^{\prime}}\right|^{2}\right\rangle n_{0}\left(1-n_{0}^{\prime}\right)\left[\nu-\nu^{\prime}\right] d^{3} p^{\prime}
$$

with the averaging over (uniform) distribution of impurities. After usual transformations, this collision integral will lead to the standard expressions for the bulk relaxation time $\tau_{i}$ and the mean free path $\mathcal{L}_{i}$.

If we want to take into account both impurity and boundary scattering simultaneously, then the effective scattering potential after the coordinate transformation will obtain the form

$$
V_{\mathrm{eff}}=U(\mathbf{R})+\delta U(\mathbf{R})+V,
$$

where $V$ is given by Eq. (10), and $\delta U$ is the small change in $U$ as a result of transformation $\mathbf{r} \rightarrow \mathbf{R}, \delta U=U(\mathbf{r})-$ $U(\mathbf{R})$.

The matrix element of this perturbation is linear in these three terms, while its square will contain, after averaging over surface inhomogeneities and distribution of bulk impurities, only the terms

$$
\begin{aligned}
\left\langle\left|V_{\text {eff }_{\mathbf{P} \mathbf{P}^{\prime}}}\right|^{2}\right\rangle= & \left\langle\left|U_{\mathbf{P} \mathbf{P}^{\prime}}\right|^{2}\right\rangle+\left\langle\left|V_{\mathbf{P P}^{\prime}}\right|^{2}\right\rangle+\left\langle\left|\delta U_{\mathbf{P P}^{\prime}}\right|^{2}\right\rangle \\
& +\left\langle\delta U_{\mathbf{P}^{\prime}}^{*} V_{\mathbf{P}^{\prime} \mathbf{P}}+\delta U_{\mathbf{P}^{\prime} \mathbf{P}} V_{\mathbf{P P}^{\prime}}^{*}\right\rangle .
\end{aligned}
$$

After the substitution into the equations for scattering probability and collision integral, the first term will reproduce the usual collision frequency with impurities $1 / \tau_{i}$, the second term will give the surface-induced collision integral from Sec. IV, while the last three terms will describe the interference between bulk and surface scattering and will be linear in both impurity concentration and surface correlator $\zeta / L$. If the impurity con- centration is small, these interference terms can be neglected, and the transport parameters will obey a simple Matthiessen's rule for independent bulk and surfaceinduced collisions,

$$
\mathcal{L}_{\text {eff }}^{-1}=\mathcal{L}_{i}^{-1}+\mathcal{L}_{\text {wall }}^{-1}
$$

where $\mathcal{L}_{\text {wall }}^{-1}$ is the surface-induced mean free path along the wall calculated in Sec. IV. Otherwise, the interference terms will complicate the picture.

The situation will be even more complicated for large concentration of impurities or beyond the Born approximation for impurity scattering. What is more important than the presence of the interference terms is that the wave functions for calculation of matrix elements should include the effects of impurity scattering. In this case, unperturbed wave functions are the attenuating plane waves, and we should include the extra terms (11) back into the perturbation $\widehat{V}$. It is obvious that the presence of strong bulk attenuation should result in the disappearance of the off-diagonal correlator $\varsigma_{12}$ from the expressions for the transport coefficients.

\section{SUMMARY}

In summary, we solved several different quantum and classical transport problems in films and channels with rough walls. We calculated the mobility (including the quantum interference corrections) and diffusion coefficients in films and channels, single-particle diffusion coefficient (a classical bouncing or billiard ball problem) in films with and without quantization of motion across the field. The results include corrections caused by the energy distortion near the walls, and are generalized to the case of particles with arbitrary energy spectrum. All of these transport coefficients are expressed explicitly via the correlation function of surface inhomogeneities. We demonstrated how the presence of surface inhomogeneities leads to a formation of a length scale, and, therefore, affects the mesoscopic effects. One of the main advantages is that our method is very straightforward, simple, and versatile.

The results allow to express the mean free path along the film, restricted by repeated reflections from the random walls, via the parameters of the correlation function of the surface inhomogeneities. This mean free path has the scales $\mathcal{L} \sim L^{2} R \ell^{-2} f(R / \lambda)$ and $\mathcal{L} \sim$ $L^{6} \ell^{-2} R^{-3} f(R / \lambda)$ in the cases without and with quantization of motion across the channel or film. The functions $f$ go to infinity in the both limits of vanishingly short and infinitely large de Broglie wavelengths $\lambda$, and has a minimum when the correlation radius of surface inhomogeneities $R \sim \lambda$. This means that the chaotization of motion by reflections from random walls can dominate over the bulk relaxation only in a finite range of particle wavelengths.

Thus, the main deficiency of the results obtained so far is that we mostly neglected bulk relaxation and collisions, and restricted ourselves to the almost ballistic regime. As a result, the mean free path along the walls was restricted only by scattering by surface inhomogeneities. Another 
interesting consequence of this assumption is that the transport coefficients contain not only the correlation of inhomogeneities from the same wall, but also the correlation of inhomogeneities from the opposite walls. Of course, this correlation function should gradually disappear from the expressions for transport coefficients with an increase in bulk relaxation. This should be associated with an reappearance of additional terms (11) in the effective Hamiltonian, as a result of distortion of the bulk wave functions by relaxation processes.

Another - and more important - anticipated effect of bulk relaxation is that the film thickness $L$ should gradually disappear from the transport coefficients being substituted by the bulk mean free path $\mathcal{L}_{\text {bulk }}$. In this case, the particles return to the wall and the wall scattering is repeated not because of reflection from the opposite wall, but as a result of bulk collisions. In the future, we plan to generalize our approach by including the effects of strong bulk relaxation; this will also give us a more realistic basis for the study of semi-infinite space with only one rough boundary.

The above results can be used for the description of transport in porous media. However, the direct application of our results is possible only for systems with very high porosity when there are practically no cavities and the width of the channels is much larger than the curvature of the walls. In this case, the correlation radius $R$ of surface inhomogeneities in the above equations has the meaning of the curvature of the pores, and the height $\ell$ is the average amplitude of the wall "oscillations."

Our method can also be applied to the study of boundary slip near rough walls. Usually, the slip effects in gases are scaled with the bulk mean free path. ${ }^{29} \mathrm{How}-$ ever, there are some experimental indications that the slip effects can contain another parameter of length. ${ }^{30}$ This additional length scale may be related to characteristics of surface inhomogeneities. ${ }^{31}$ Our method will allow one to relate this new contribution to the boundary slip to the correlation function of surface inhomogeneities, namely, to the length scale $\left(\ell^{2} R\right)^{1 / 3}$. Our preliminary results show ${ }^{32}$ that this calculation will also require the simultaneous analysis of the effects of surface roughness and bulk relaxation.

Another option for continuation of this work is the modification of the boundary condition $\Psi=0$ on the walls. Such a boundary condition means an infinite barrier for particles (waves) at the boundary. This can be changed to a finite barrier. The results will describe the diffusion in layered media and the transmission and reflection coefficients for layers with rough walls.

The results of this paper can give an alternative algorithm for numerical study of the bouncing ball problem (a billiard model, Sec. V). There are some numerical indications that the asymptotic motion of such a bouncing particle along the rough wall deviates from the standard diffusion behavior $l \propto D t^{2}$. At present, it is not completely clear whether this deviation is an intrinsic property of the problem, or a result of insufficient accuracy in treating the boundary problem. Our method can simplify the computations by reducing the computational problem of scattering by a random wall to an equivalent, but much more transparent computationally, bulk problem in the potentials (10), (12) or with an effective mass (16). If the deviation from a diffusive asymptotic will persist, this will mean that either the Hamiltonian (10) has some very special properties which are different from other random potentials, or that the diffusion chaotization of motion in a random bulk potential is even more nontrivial than is usually assumed.

\section{ACKNOWLEDGMENTS}

We are grateful to $\mathrm{K}$. Musaelian for valuable discussions in the initial stage of this work, and to $\mathrm{Z}$. Tesanovic for acquainting us with several important publications in this field. This work was supported by NSF Grant No. DMR-9412769.
${ }^{1}$ A.V. Chaplik and M.V. Entin, Zh. Eksp. Teor. Fiz. 55, 990 (1968) [Sov. Phys. JETP 28, 514 (1969)].

${ }^{2}$ L.A. Falkovskii, Zh. Eksp. Teor. Fiz. 58, 1830 (1970) [Sov. Phys. JETP 31, 981 (1970)]; 33, 454 (1971) [60, 838 (1971)].

${ }^{3}$ F.G. Bass and I.M. Fuks, Wave Scattering from Statistically Rough Surfaces (Pergamon, New York, 1979).

${ }^{4}$ J.A. DeSanto and G.S. Brown, Analytical Techniques for Multiple Scattering from Rough Surfaces, edited by E. Wolf, Progress in Optics Vol. XXIII (North-Holland, Amsterdam, 1986).

${ }^{5}$ J.R. Willis, in Mathematic Modeling in Non-Destructive Testing, edited by M. Blakemore and G.A. Georgiou (Clarendon, Oxford 1988), p. 57.

6 J.A. Ogilvy, Theory of Wave Scattering from Random Surfaces (Adam Hilger, Bristol, 1991).

${ }^{7}$ Interactions of Atoms and Molecules with Solid Surfaces, edited by V. Bortolani, N.H. March, and M.P. Tosi (Plenum, New York, 1990).
${ }^{8}$ E.I. Thorsos and D.R. Jackson, J. Acoust. Soc. Am. 86, 261 (1989).

${ }^{9}$ Z. Tesanovic, M.V. Jaric, and S. Maekawa, Phys. Rev. Lett. 57, 2760 (1986).

${ }^{10}$ R. Lenk and A. Knabchen, J. Phys. Condens. Matter 5, 6563 (1993).

${ }^{11}$ N. Trivedi and N.W. Ashcroft, Phys. Rev. B 38, 12298 (1988).

12 P.M. Levy, S. Zhang, and A. Fert, Phys. Rev. Lett. 65, 1643 (1990).

${ }^{13}$ A.C. Ehrlich, Phys. Rev. Lett. 71, 2300 (1993).

${ }^{14}$ G. Reiss, E. Hastreiter, H. Bruckl, and J. Vancea, Phys. Rev. B 43, 5176 (1991).

15 J.A. Konrady, Jr., J. Acoust. Soc. Am. 56, 1687 (1974).

${ }^{16}$ H.D.I. Abrabanel, J. Acoust. Soc. Am. 68, 1459 (1980).

${ }^{17}$ R.E. Goldstein, A.I. Pesci, and V. Romero-Rochin, Phys. Rev. A 41, 5504 (1990).

${ }^{18}$ A.B. Isers, A.A. Puzenko, and I.M. Fuks, J. Electromag. Waves Appl. 5, 1419 (1991). 
${ }^{19}$ A.E. Meyerovich and S. Stepaniants, Phys. Rev. Lett. 73, 316 (1994).

${ }^{20}$ A.E. Meyerovich and S. Stepaniants, Phys. Rev. B 49, 3400 (1994).

${ }^{21}$ A.E. Meyerovich and B.E. Meyerovich, Sov. Phys. JETP 66, 833 (1987).

${ }^{22}$ B.G. Orr, H.M. Jaeger, A.M. Goldman, and C.G. Kuper, Phys. Rev. B 32, 7586 (1985).

${ }^{23}$ C.W.J. Beenakker and H. van Houten, Phys. Rev. Lett. 63, 1857 (1989).

${ }^{24}$ V.I. Kozub and A.A. Krokhin, J. Phys. Condens. Matter 5, 9135 (1993).

${ }^{25}$ K. Nakazato and R.J. Blaikie, J. Phys. Condens. Matter 3, 5729 (1991).
${ }^{26}$ P.A. Lee and T.V. Ramakrishnan, Rev. Mod. Phys. 57, 287 (1985)

${ }^{27}$ B. Altshuler, in Nanostructures and Mesoscopic Systems, edited by W.P. Kirk and M.A. Reed (Academic Press, New York, 1991), pp. 405-416.

${ }^{28}$ C. Itzykson and J.-M. Drouffe, Statistical Field Theory (Cambridge University Press, Cambridge 1991), Vol. 2, Chap. 10.

${ }^{29}$ E.M. Lifshitz and L.P. Pitaevski, Physical Kinetics (Plenum, New York, 1979), Sec. 1.14.

${ }^{30}$ D.A. Ritchie et al., Phys. Rev. Lett. 59, 465 (1987).

${ }^{31}$ D. Einzel, P. Panzer, and M. Liu, Phys. Rev. Lett. 64, 2269 (1990).

${ }^{32}$ K. Ivanova-Moser and A.E. Meyerovich (unpublished). 